\title{
Costos hospitalarios directos de la neumonía asociada al ventilador
}

\section{Hospital direct costs of ventilator-associated pneumonia}

\author{
Alex García, Nelson Fonseca, Nelson Giraldo, Bladimir Gil, \\ Ana Pamplona, Ana Diaz • Medellín (Colombia)
}

\section{Resumen}

Objetivo: el objetivo del estudio fue determinar los costos directos de tratar a los pacientes mecánicamente ventilados que desarrollan un episodio de neumonía asociada al ventilador desde la perspectiva del hospital.

Diseño: entre junio $1^{\circ}$ de 2011 y junio $1^{\circ}$ de 2012,90 pacientes en ventilación mecánica por más de 48 horas en tres unidades de cuidados intensivos medicoquirúrgicas fueron evaluados para la presencia de neumonía asociada al ventilador.

Se determinaron los costos de estancia en la unidad, antibióticos, estudios imagenológicos y microbiológicos. Se determinó el costo total en ventilación mecánica con neumonía asociada al ventilador y sin neumonía. Se estableció el costo incremental de un episodio de neumonía. Los costos se tasaron en pesos colombianos del año 2011 y se convirtieron a dólares 2012.

Resultados: 90 pacientes, 33 pacientes tuvieron neumonía asociada al ventilador. El costo promedio por paciente en ventilación mecánica fue 7950 dólares. El costo promedio por paciente en ventilación mecánica con neumonía asociada al ventilador fue 21217 dólares. El costo incremental fue 14328 dólares $(\mathrm{p}<0.001)$.

La fuente de los costos fue $69 \%$ en estancia, $21 \%$ en el tratamiento antibiótico, $6 \%$ en estudios de laboratorio e imagenológicos, y $1 \%$ en estudios microbiológicos.

En el análisis multivariado por regresión lineal múltiple la presencia de neumonía asociada al ventilador se asoció significativamente con los costos totales $(\mathrm{p}=0.0001)$

Conclusiones: la neumonía asociada al ventilador incrementó los costos totales. Los pacientes con neumonía asociada al ventilador tuvieron un costo adicional de 14328 dólares. (Acta Med Colomb 2014: 39: 238-243)

Palabras clave: cuidados intensivos, neumonía asociada al ventilador, costos en salud, pacientes críticamente enfermos, sepsis.

\footnotetext{
Abstract

Objective: the aim of the study was to determine the direct costs of treating mechanically ventilated patients who develop an episode of ventilator-associated pneumonia from the hospital perspective.

Design: between June 1, 2011 and June 1, 2012, 90 patients on mechanical ventilation for more than 48 hours in 3 medical-surgical units of intensive care were evaluated for the presence of ventilatorassociated pneumonia.

Costs of unit stay, antibiotics, imaging and microbiological studies were determined. The total cost of mechanical ventilation with ventilator-associated pneumonia and without pneumonia was determined. The incremental cost of an episode of pneumonia was established. Costs were calculated according to the value of Colombian pesos in 2011 and converted to dollars valued in 2012.

Results: from a total of 90 patients, 33 had ventilator-associated pneumonia.

The average cost per patient on mechanical ventilation was $\$ 7950$. The average cost per patient on mechanical ventilation with ventilator-associated pneumonia was \$21217. The incremental cost was \$ 14328 ( $\mathrm{p}<0.001)$.

The source of the costs was $69 \%$ in hospital stay, $21 \%$ in antibiotic treatment, $6 \%$ in laboratory studies and imaging, and $1 \%$ in microbiological studies.
}

Dr. Alex García: Departamento de Anestesiología y Medicina Crítica, Escuela de Medicina, Universidad de Antioquia. Departamento de Cuidados Intensivos, Hospital Pablo Tobón Uribe. Unidad de Terapia Intensiva Adultos, Clínica Universitaria Bolivariana; Dr. Nelson Fonseca: Programa de Medicina Crítica y Cuidado Intensivo. Facultad de Medicina, Universidad CES. Facultad de Medicina, Universidad Pontificia Bolivariana; Dr. Nelson Giraldo: Departamento de Cuidados Intensivos, Hospital Pablo Tobón Uribe; Dr. Bladimir Gil: Unidad de Cuidados Intensivos, Clínica Las Américas; Dras. Ana Pamplona y Ana Diaz: Semillero de Investigación Facultad de Medicina, Médicas Universidad Pontifica Bolivariana, Escuela de Medicina. Medellín (Colombia).

Correspondencia: Dr. Alex García. Medellín (Colombia)

E-mail: deorumalex@yahoo.com.ar Recibido: 16/VII/2013 Aprobado: 1/VIII/2014 
In the multivariate analysis by multiple linear regression, the presence of ventilator-associated pneumonia was significantly associated with the total costs $(\mathrm{p}=0.0001)$.

Conclusions: ventilator-associated pneumonia increased total costs. Patients with ventilatorassociated pneumonia had an additional cost of \$ 14 328. (Acta Med Colomb 2014: 39: 238-243)

Keywords: intensive care, ventilator-associated pneumonia, health care costs, critically ill patients, sepsis.

\section{Introducción}

Las infecciones asociadas al cuidado de la salud (IAH) son frecuentes en las unidades de cuidados intensivos (UCI) (1). La neumonía nosocomial es la segunda causa de infección adquirida en el hospital y la primera en mortalidad (2). Es conocido su efecto en la mortalidad y en la morbilidad (3). Sin embargo, el impacto económico de esta infección en el paciente, el hospital, el sistema de salud y la sociedad no ha sido adecuadamente establecido $(4,5)$.

En un estudio retrospectivo de casos y controles, Rello y colaboradores en 1998, analizaron 9080 pacientes en Estados Unidos, de los cuales 842 desarrollaron neumonía asociada a la ventilación mecánica (NAV). El desarrollo de NAV se asoció a un aumento de los costos hospitalarios en promedio de 40000 dólares por paciente (6).

Dietrich y colaboradores en el 2002 en un hospital universitario en Alemania, analizaron 48 pacientes con neumonía y 66 controles. Los pacientes con neumonía tuvieron mayor estancia en la UCI y de hospitalización. El costo adicional de los pacientes con neumonía fue de 14606 dólares (7).

Warren y colaboradores en 2003 en una UCI médica y quirúrgica determinaron los costos atribuibles a la neumonía en pacientes ventilados mecánicamente por más de 24 horas. El costo atribuible por un episodio de NAV fue 11 897 dólares (8).

Stephane Hugonnet y colaboradores en 2004 en una UCI médica del Hospital Universitario de Ginebra, realizaron un estudio de casos y controles para determinar la utilización de recursos y costos de los pacientes con NAV. De 441 pacientes en ventilación mecánica por más de 48 horas, 106 tuvieron NAV (24\%). Los pacientes con NAV tuvieron más días de ventilación mecánica, mayor estancia en la UCI y el costo promedio atribuible por episodio de neumonía fue de 10450 dólares (9).

En 2005 Safdar y colaboradores, realizaron una revisión sistemática de la literatura para determinar la incidencia de NAV, la estancia hospitalaria y los costos. Encontraron que un $20 \%$ de los pacientes que reciben ventilación mecánica por más de 48 horas desarrollaron NAV. Los pacientes con NAV tuvieron una mayor estancia en la UCI, en promedio 6.10 días adicionales y tuvieron incremento en los costos hospitalarios totales en 10019 dólares (10).

Estos estudios aportan información relevante en pases desarrollados; sin embargo, hay información insuficiente para los países en vía de desarrollo sobre los recursos utilizados en la atención de los pacientes en ventilación mecánica que presentan un episodio de NAV y de los costos atribuidos a la NAV.

El objetivo de este estudio es estimar los recursos empleados y los costos directos totales de atención de los pacientes en ventilación mecánica que desarrollan un episodio de neumonía asociada al ventilador desde el punto de vista del hospital.

\section{Pacientes y métodos}

El estudio se realizó en tres unidades de cuidados intensivos medicoquirúrgicas de carácter universitario en Medellín, Colombia. Fue aprobado por los comités de ética de las instituciones participantes, y por tratarse de un estudio de no intervención, según la legislación de nuestro país, no fue necesario solicitar consentimiento informado a los pacientes o sus familiares (11). Ni los investigadores, ni las instituciones participantes recibieron compensación económica o fondos por la realización del estudio.

Se incluyeron todos los pacientes mayores de 18 años, hospitalizados en UCI, con más de 48 horas en ventilación mecánica. Se realizó seguimiento prospectivo a todos los pacientes que ingresaron desde el primero de junio de 2011 hasta el primero de junio de 2012 para determinar la aparición de NAV. El diagnóstico de NAV se realizó con base en los criterios del CDC y para el aislamiento microbiológico se utilizó aspirado traqueal cuantitativo con punto de corte de $10^{5} \mathrm{cfu} / \mathrm{mL}$. (12). El manejo de los pacientes fue a discreción de los médicos tratantes según los protocolos institucionales.

Se excluyeron pacientes embarazadas, inmunosuprimidos, posoperatorio de trasplante de órganos sólidos, con órdenes de no reanimación o con limitación del esfuerzo terapéutico y con infecciones de tratamiento prolongado como peritonitis, infección intraabdominal complicada, y mediastinitis.

El seguimiento se realizó registrando las variables en un formulario previamente diseñado. Para todos los pacientes se registraron las variables demográficas: edad, sexo, diagnóstico de ingreso a la UCI, puntaje Acute Physiology and Chronic Health Evaluation scoring system (APACHE) versión II, comorbilidades como hipertensión arterial (HTA), diabetes mellitus (DM), enfermedad pulmonar obstructiva crónica (EPOC), enfermedad coronaria, falla cardiaca, insuficiencia renal crónica (IRC) y neoplasias. También se registró la duración total de la ventilación mecánica, la presencia de NAV, los días estancia en la UCI y en el hospital y la mortalidad al egreso de la UCI y del hospital. 
Se registraron los recursos empleados en la atención de cada paciente en ventilación mecánica. Se determinaron los días estancia en la UCI desde el día de ingreso hasta la salida. Se registró la cantidad total de los estudios imagenológicos realizados que incluyeron radiografía de tórax, ecografía de tórax y tomografía de tórax. Los estudios microbiológicos incluyeron los cultivos de aspirado traqueal, lavado broncoalveolar, urocultivo y hemocultivos. Los estudios de laboratorio incluyeron gases arteriales, hemoleucograma y proteína $\mathrm{C}$ reactiva (PCR). Para establecer el valor de los costos; a cada recurso utilizado se le asignaron los precios del manual del Seguro Obligatorio para Accidentes de Tránsito (SOAT) colombiano actualizado al año 2011, que es un manual de vigencia nacional que establece los precios de atención hospitalaria y sirve de base para que el hospital elabore la factura de cobro al pagador y de "FARMAPRECIOS"que es un listado actualizado de productos farmacéuticos disponibles en Colombia (13). Los costos totales se obtuvieron de la suma de todos los costos anteriores y se tasaron en dólares americanos teniendo en cuenta la tasa oficial de cambio para junio de 2012. Los costos de los pacientes con NAV fueron comparados con los costos de los pacientes sin NAV.

\section{Análisis estadístico}

El desenlace primario fue la diferencia de los promedios del costo total de los pacientes con y sin NAV. Se planeó una muestra total de al menos 31 pacientes en cada grupo, para buscar una diferencia en el promedio de 8 millones (equivalente US \$4500) con una desviación estándar de 11 millones considerando un error alfa de 0.05 , un error beta de 0.2 y una pérdida de $10 \%$ en cada grupo, con base en estudios previos $(3,8)$.

Se presentan las variables continuas con distribución normal como medias \pm desviación estándar y como medianas

Tabla 1. Características basales.

\begin{tabular}{|c|c|c|c|}
\hline & $\underset{\mathbf{n}=\mathbf{5 7}}{\operatorname{Sin} \mathbf{N A V}}$ & $\begin{array}{c}\text { Con NAV } \\
n=33\end{array}$ & $\mathbf{P}$ \\
\hline Edad en años, mediana (RIQ) & $55(41.5-70.5)$ & $64(50-80)$ & 0.063 \\
\hline Sexo masculino $(\%)$ & 44.3 & 55.6 & 0.38 \\
\hline APACHE II mediana (RIQ) & $17(12-21)$ & $22(14-27)$ & 0.03 \\
\hline Comorbilidades (\%) & $\begin{array}{l}\text { HTA ( 45.9) } \\
\text { EPOC ( 6.6) }\end{array}$ & $\begin{array}{l}\text { HTA (62.9) } \\
\text { EPOC ( 5.7) }\end{array}$ & \\
\hline Dx ingreso UCI (\%) & $\begin{array}{c}\text { Sepsis (27.9) } \\
\text { ECV (16.4) } \\
\text { Trauma (13.1) } \\
\text { Cx mayor (13.1) }\end{array}$ & $\begin{array}{c}\text { Sepsis }(22.2) \\
\text { ECV (19.5) } \\
\text { Trauma }(16.7) \\
\text { Cx mayor }(11.1)\end{array}$ & 0.52 \\
\hline \multicolumn{4}{|c|}{$\begin{array}{l}\text { NAV: neumonía asociada al ventilador } \\
\text { HTA: hipertensión arterial } \\
\text { EPOC: enfermedad pulmonar obstructiva crónica } \\
\text { ECV: enfermedad cerebrovascular } \\
\text { Cx: cirugía } \\
\text { RIQ rango intercuartil }\end{array}$} \\
\hline
\end{tabular}

con rango intercuartil las que no tienen distribución normal. Las variables categóricas se presentan como porcentajes. La comparación de las variables continuas entre los dos grupos de estudio se realizó con la prueba t de Student, cuando tuvieron distribución normal o la prueba de rangos de Wilcoxon, cuando no presentaron distribución normal. La comparación de las variables categóricas se hizo con la prueba de Chi cuadrado, o con la prueba exacta de Fisher cuando se requirió por el tamaño de la muestra. Se consideró estadísticamente significativo un valor de $\mathrm{p}$ de dos colas menor de 0.05 .

Se hizo análisis multivariado para determinar la significancia de la NAV en el costo total, por medio de una regresión lineal múltiple, donde los costos totales de hospitalización fue la variable dependiente y las variables independientes las encontradas con significación clínica según la revisión de la literatura y el análisis univariado con una $\mathrm{p}<0.2 \mathrm{El}$ análisis estadístico se realizó con el programa STATA versión 12.1

\section{Resultados}

Durante el periodo de estudio 90 pacientes en ventilación mecánica por más de 48 horas fueron evaluados. De éstos, 33 pacientes presentaron NAV (36.7\%) y 57 no la presentaron. Las neumonías tempranas, es decir, antes del día cinco de ventilación mecánica, fueron $9.1 \%$ y el resto fueron NAV tardías (90.9\%). Los gérmenes más frecuentemente asilados fueron Klebsiella pneumoniae (25\%), Pseudomonas aeruginosa $(17.8 \%)$ y $S$. aureus sensible a meticilina $(10.7 \%)$.

Los pacientes con NAV tuvieron mayor severidad demostrada por el puntaje APACHE II y mayor edad que el grupo sin NAV. Las características basales de cada grupo son presentadas en la Tabla 1.

Los días estancia y los días de ventilación mecánica fueron significativamente menores en el grupo sin NAV y la mortalidad hospitalaria también fue menor, pero sin alcanzar significancia estadística (Tabla 2).

Los pacientes con NAV tuvieron tres veces más estudios microbiológicos, dos veces más estudios de laboratorio e imagenológicos (Tabla 3). Los pacientes con NAV tuvieron más antibióticos prescritos. Los antibióticos más utilizados fueron piperacilina tazobactam, cefepime y meropenem.

Tabla 2. Desenlaces clínicos.

\begin{tabular}{|l|c|c|c|}
\hline Variable & $\begin{array}{c}\text { Pacientes } \\
\text { sin NAV } \\
\mathbf{n = 5 7}\end{array}$ & $\begin{array}{c}\text { Pacientes } \\
\text { con NAV } \\
\mathbf{n = 3 3}\end{array}$ & P \\
\hline $\begin{array}{l}\text { Días de ventilación } \\
\text { mecánica }\end{array}$ & $\begin{array}{c}6 \\
\text { (RIQ 4-11.5) }\end{array}$ & (RIQ 15.5-34.5) & $<0.001$ \\
\hline Días de estancia en UCI & $11.6( \pm 7.8)$ & $31.97( \pm 20.84)$ & $<0.001$ \\
\hline Mortalidad hospitalaria & $21.1 \%$ & $31.3 \%$ & $\mathrm{p}=0.41$ \\
\hline \multicolumn{2}{|l}{ NAV: neumonía asociada a la ventilación mecánica. } \\
RIQ: rango intercuartil. UCI: unidad de cuidados intensivos \\
\hline
\end{tabular}


Tabla 3. Recursos utilizados en pacientes con neumonía asociada al ventilador.

\begin{tabular}{|c|c|c|c|c|}
\hline Variable & $\begin{array}{c}\text { No NAV } \\
\text { Media (Desviación estándar) }\end{array}$ & $\begin{array}{c}\text { Si NAV } \\
\text { Media (Desviación estándar) }\end{array}$ & $\begin{array}{l}\text { Diferencia de medias } \\
\text { (IC 95\%) }\end{array}$ & Valor de $\mathrm{p}$ \\
\hline Total de cultivos & $3.73(0.54)$ & $9.75(0.67)$ & $-6.02(-7.76 \mathrm{a}-4.27)$ & $<0.0001$ \\
\hline Total de imágenes & $7.12(0.68)$ & $15.39(1.43)$ & $-8.27(-11.08$ a -5.45$)$ & $<0.0001$ \\
\hline Total paraclínicos & $33.71(2.71)$ & $81.75(6.15)$ & $-48.03(-59.71$ a -36.36$)$ & $<0.0001$ \\
\hline
\end{tabular}

Tabla 4. Resumen de los costos promedio discriminados por origen (en pesos).

\begin{tabular}{|l|c|c|c|}
\hline & $\begin{array}{c}\text { Pacientes } \\
\text { sin NAV }\end{array}$ & $\begin{array}{c}\text { Pacientes } \\
\text { con NAV }\end{array}$ & P \\
\hline Estancia UCI & 10206250 & 28373106 & $<0.001$ \\
Antibióticos & 3204638 & 7842064 & $<0.001$ \\
Estudios imagenológicos & 552900 & 1211290 & $<0.001$ \\
Estudios microbiológicos & 150071 & 417850 & $<0.001$ \\
Exámenes de laboratorio & 872650 & 2149633 & $<0.001$ \\
\hline Totales & $\mathbf{1 4 9 8 6 5 1 0}$ & $\mathbf{3 9 9 9 3 9 4 5}$ & $<\mathbf{0 . 0 0 1}$ \\
\hline NAV: neumonía asociada a la ventilación mecánica. \\
UCI: Unidad de Cuidados Intensivos
\end{tabular}

Los costos de hospitalización, imagenología, estudios de laboratorio y microbiológicos y antibióticos fue significativamente mayor en el grupo de NAV (Tabla 4). El costo total promedio de los pacientes sin NAV fue US\$ 7950 vs US\$ 21217 de los pacientes con NAV, $(\mathrm{p}<0.001)$. El costo incremental fue US\$ 14 328. Cuando se discriminó por origen de los costos, se encontró que $69 \%$ fue por hospitalización, $21 \%$ por antibióticos, $6 \%$ exámenes y $1 \%$ cultivos microbiológicos.

Se realizó un análisis multivariado por regresión lineal múltiple y se encontró que la presencia de NAV se asoció significativamente con los costos totales (Tabla 5). En la regresión lineal múltiple, el costo total de la hospitalización depende en mayor parte de la estancia en UCI y en segundo lugar del uso de los antibióticos. Ambas variables son afectadas por la presencia de NAV.

\section{Discusión}

El principal hallazgo de este estudio es que la NAV incrementó significativamente los recursos utilizados y los costos totales de atención de los pacientes y que este costo incremental fue en promedio de 14000 dólares por episodio. Los principales determinantes del costo fueron la estancia en la unidad de cuidados intensivos y el uso de antibióticos. Adicionalmente los pacientes con NAV también incurrieron en mayores costos por estudios de laboratorio clínico, microbiológico e imagenológicos.

Los pacientes con NAV estuvieron más días en ventilación mecánica y tuvieron una estancia en la UCI más prolongada; la diferencia promedio en nuestro estudio fue de 14 días. Esto es importante en la medida que la estancia en la UCI fue el principal determinante del costo total de los pacientes.

La mortalidad fue mayor en los pacientes con neumonía aunque no alcanzó significancia estadística; esto también es consistente con un estudio reciente en el que la neumonía asociada al ventilador aumentó la morbilidad pero no la mortalidad (6).

Tabla 5. Regresión lineal múltiple.

\begin{tabular}{|c|c|c|c|c|c|c|c|}
\hline \multirow[t]{2}{*}{ Costo total } & \multirow{2}{*}{$\begin{array}{l}\text { Coeficiente } \\
\text { beta }\end{array}$} & \multirow{2}{*}{$\begin{array}{l}\text { Coeficiente beta } \\
\text { estandarizado }\end{array}$} & \multirow[t]{2}{*}{ Error estándar } & \multirow[t]{2}{*}{ Valor $\mathbf{t}$} & \multirow[t]{2}{*}{ Valor $\mathbf{p}$} & \multicolumn{2}{|c|}{ Intervalo de confianza $95 \%$} \\
\hline & & & & & & Inferior & Superior \\
\hline Días UCI & 919384.5 & .7568985 & 4207.111 & 218.53 & 0.000 & 911016.7 & 927752.2 \\
\hline NAV & 314198.8 & .0073101 & 127884.6 & 2.46 & 0.016 & 59841.48 & 568556.2 \\
\hline Costo antibióticos & 1.019663 & .3096295 & .0087447 & 116.60 & 0.000 & 1.00227 & 1.037055 \\
\hline Costo cultivos & 1.575623 & .015951 & .2885133 & 5.46 & 0.000 & 1.001781 & 2.149464 \\
\hline Costo imágenes & 1.098415 & .0312861 & .1101609 & 9.97 & 0.000 & .87931 & 1.317521 \\
\hline Constante & 302168.9 & & 79902.86 & 3.78 & 0.000 & 143245.4 & 461092.5 \\
\hline
\end{tabular}

Supuestos cumplidos linealidad y no hay multicolinealidad (con el factor inflación de varianza) y la independencia de los residuales, supuestos incumplidos: homogeneidad de varianza y normalidad de los residuales. 


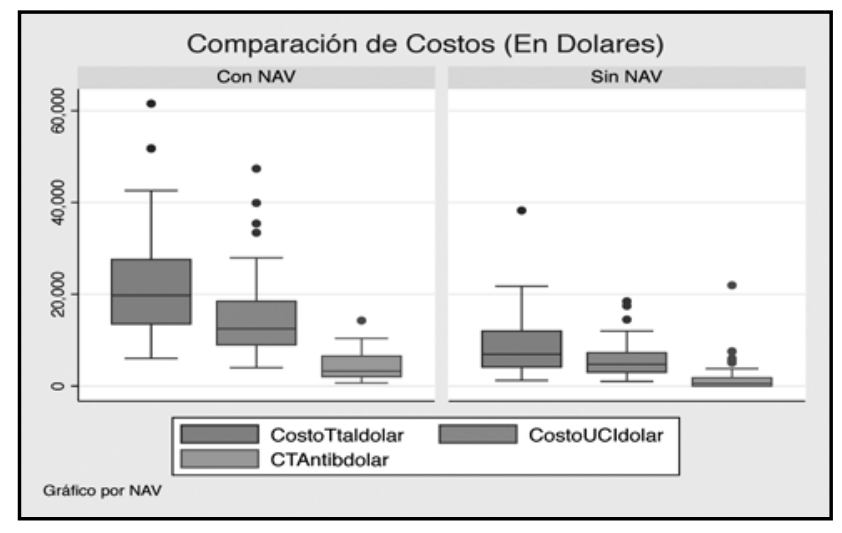

Figura 1. Comparación de los costos en dólares de los pacientes sin neumonía asociada al ventilador y de los pacientes con neumonía asociada al ventilador.

a. CTAntibdolar: antibióticos, costos de los antibióticos prescritos.

b. CostoTtaldolar: costos totales, incluye los costos de estancia en la UCI, costos de antibióticos, estudios microbiológicos e imagenológicos.

c. CostoUCIdolar: costos en UCI, costos correspondientes a los días de estancia en la UCI.

Nuestros resultados están en consonancia con estudios previos publicados, donde la NAV aumentó el costo total de atención. Rello y colaboradores encontraron que el desarrollo de NAV se asoció a un aumento de los costos hospitalarios en promedio de 40000 dólares por paciente (6). El diagnóstico de NAV se basó en los códigos de diagnóstico del egreso; y los costos fueron determinados por la factura del hospital. A diferencia de nuestro estudio no se especificó la distribución de los costos utilizados en los pacientes con NAV.

Dietrich analizó los datos de 114 pacientes, $50 \%$ de éstos con enfermedad neurológica como causa de ingreso a la UCI. Los pacientes con neumonía tuvieron significativamente mayor estancia en la UCI y en el hospital. El costo adicional de los pacientes con neumonía fue de 14606 dólares. Los pacientes con neumonía tuvieron dos veces más costos por antibióticos y estudios microbiológicos que los pacientes sin neumonía. En nuestro estudio el principal diagnóstico de ingreso a la UCI fue sepsis, lo que sugiere que la NAV puede aumentar los costos de una manera similar en distintas poblaciones de pacientes.

David Warren y colaboradores encontraron que los pacientes con NAV tuvieron más días de ventilación mecánica y mayor estancia en la UCI. El costo atribuible por un episodio de NAV fue 11897 dólares (8). En este estudio los principales determinantes del costo fueron la estancia en la UCI, y los costos de farmacia. Nuestro estudio también encontró que la estancia y los antibióticos son los principales determinantes de los costos totales, pero también encontramos diferencias en los costos de laboratorio, microbiológicos e imagenológicos. Esto puede explicarse por los costos del proceso diagnóstico, ya que la sospecha de NAV implica la toma de cultivos, radiografía de tórax y muchas veces el inicio empírico de antibióticos, lo que implica un consumo de estos recursos.

Las fortalezas de nuestro estudio están en su diseño prospectivo y en la inclusión de todos los recursos importantes consumidos que pueden influenciar el costo. Además se tuvo en cuenta la exclusión de variables que pudieran afectar la estancia en la unidad de cuidados intensivos y la duración de la ventilación mecánica independiente de la NAV. La consistencia de nuestros resultados con los estudios previamente publicados en Estados Unidos y en Europa, nos permite concluir que a pesar de las diferentes poblaciones de pacientes, de protocolos de manejo y sistemas de salud, la NAV impone una carga económica similar tanto en países desarrollados como en vía de desarrollo, siendo para estos últimos más onerosa en vista de la mayor limitación de recursos.

La limitación principal del estudio es que existe una relación de reciprocidad entre la aparición de NAV y el aumento de la estancia, a su vez este aumento de la estancia es el principal determinante de los costos totales. ¿Entonces es la NAV la que directamente aumenta los costos, o es indirectamente a través de aumento de la estancia? Para tratar de resolver este punto nosotros tomamos los días de estancia desde el inicio de la ventilación mecánica, lo que nos permitió demostrar que la aparición de NAV implicó un aumento en el consumo de este recurso y el análisis de regresión lineal múltiple corroboró que los días estancia y el consumo de antibióticos son dependientes de la presencia de NAV.

Otra limitación es las diferencias en las características demográficas entre los dos grupos especialmente en severidad y comorbilidades, lo que pudiera haber influenciado la cantidad de recursos utilizados y por ende los costos. Como no se realizó aleatorización, no fue posible controlar estas diferencias sociodemográficas; y realmente es difícil diferenciar por las características del estudio si estas diferencias en severidad y comorbilidades fueron un factor de riesgo para la aparición de NAV y secundariamente a ésta se incrementaron los recursos gastados y los costos. Sin embargo, en el análisis multivariado nosotros encontramos que la aparición de NAV fue la variable principal que determinaba la utilización de recursos.

En esta evaluación económica parcial, nosotros identificamos los recursos hospitalarios utilizados en el diagnóstico y tratamiento de los pacientes en ventilación mecánica que presentan un episodio de neumonía asociada al ventilador, pero para valorizar estos recursos utilizamos los precios del manual SOAT, ya que es un manual tarifario nacional, con la intención de que la estimación de los costos pudiera extrapolarse a todo el país, además que en algunas ocasiones sirve de base para negociar tarifas con los pagadores. No cuantificamos los costos de personal tanto médico y de enfermería, porque aunque es evidente que los pacientes con NAV requieren mayor inversión de tiempo de personal y consumo de este recurso, es difícil de cuantificar, y además nuestro propósito era determinar el costo incremental comparado con los pacientes en ventilación mecánica sin NAV, y para el hospital en el momento de cancelar este recurso no existe ninguna diferencia y por tanto desde esta perspectiva no sería un costo relevante. 
La estimación de los costos de las infecciones nosocomiales tiene importancia no sólo para el hospital, sino también para apreciar el impacto económico que estas infecciones pueden tener en el sistema de salud. En nuestro país el sistema de vigilancia epidemiológica GRUVECO, recoge información de las unidades de cuidados intensivos de todo el país (2). En el informe más reciente publicado, que comprende el periodo 2007-2009 se reportaron 858 episodios de NAV. Tomando como base nuestros resultados, la NAV implicó un costo para nuestro sistema de salud de 12 millones de dólares. Estos datos nos permiten apreciar el potencial de ahorro en recursos y dinero de las medidas preventivas y de motivación para los hospitales en su implementación, ya que además de estar mejorando la calidad de atención de los pacientes, están conservando una importante cantidad de recursos y dinero. Hacia el futuro, hay un gran interés en disminuir la NAV a casi cero (14) y para este objetivo, se deben realizar estudios sobre el impacto económico de la implementación de estas estrategias como los paquetes o "bundles" para prevenir la NAV (15), los protocolos de suspensión de la sedación y de destete ventilatorio (5). También se deben evaluar la costo-efectividad de implementación de nuevas tecnologías como los tubos de succión subglótico continua (16-19).

En conclusión la neumonía asociada al ventilador aumentó la utilización de recursos y el costo de los pacientes en ventilación mecánica. El aumento de los costos fue en promedio de 25 millones de pesos por episodio.

\section{Declaración de fuentes de financiación y conflicto de intereses}

Todos los autores declaramos que no tenemos ningún conflicto de interés en el desarrollo de esta investigación, que no hemos recibido fondos de ningún tipo y que la investigación se ajustó a los protocolos del comité de ética de cada institución participante, a la legislación vigente en nuestro país y a la Declaración de Helsinki de 1975.

\section{Referencias}

1. Landrigan CP, Parry GJ, Bones CB, Hackbarth AD, Goldmann D a, Sharek PJ. Temporal trends in rates of patient harm resulting from medical care. $N$ Engl $J$ Med 2010; 363(22): 2124-34.
2. Molina F, Fonseca N, Jaramillo C, Mejía S, Arango J, Benitez F, et al. Epidemiologia de las infecciones nosocomiales asociadas a dispositivos en 35 unidades de cuidados intensivos de Colombia (2007-2008). Acta Colomb Cuid Intensivo 2009; 9(1): 9-23.

3. Safdar N, Crnich CJ, Maki DG. The pathogenesis of ventilator-associated pneumonia: its relevance to developing effective strategies for prevention. Respir Care 2005; 50(6): 725-39.

4. Torres A, Ewig S, Lode H, Carlet J. Defining, treating and preventing hospital acquired pneumonia: European perspective. Intensive Care Med. 2009 Jan;35(1):9-29.

5. Muscedere J, Dodek P, Keenan S, Fowler R, Cook D, Heyland D. Comprehensive evidence-based clinical practice guidelines for ventilator-associated pneumonia: prevention. J Crit Care 2008; 23(1): 126-37.

6. Rello J. Epidemiology and Outcomes of Ventilator-Associated Pneumonia in a Large US Database. Chest 2002; 122(6): 2115-21.

7. Dietrich ES, Demmler M, Schulgen G, Fekec K, Mast O, Pelz K, et al. Nosocomial Pneumonia: A Cost-of-Illness Analysis. Infection 2002; 30(2): 61-7.

8. Warren DK, Shukla SJ, Olsen M a, Kollef MH, Hollenbeak CS, Cox MJ, et al. Outcome and attributable cost of ventilator-associated pneumonia among intensive care unit patients in a suburban medical center. Crit Care Med 2003; 31(5): 1312-7.

9. Hugonnet S, Eggimann P, Borst F, Maricot P. Impact of ventilator-associated pneumonia on resource utilization and patient outcome. Infect Control 2004; 25(12): 1-8.

10.Safdar N, Dezfulian C, Collard HR, Saint S. Clinical and economic consequences of ventilator-associated pneumonia: A systematic review. Crit Care Med 2005; 33(10): 2184-93.

11. Colombia M de S. Resolucion \# 008430 de 1993. Por la cual se Establ. las normas científicas, técnicas y Adm. para la Investig. en salud. 1993. p. 1-12.

12.Horan TC, Andrus M, Dudeck M a. CDC/NHSN surveillance definition of health care-associated infection and criteria for specific types of infections in the acute care setting. Am J Infect Control 2008; 36(5): 309-32.

13.FARMAPRECIOS [Internet]. 2011 [cited 2012 Jul 7].p. 1. Available from: http:// www.plmfarmacias.com/colombia/farmaprecios/Productos.aspx

14. Klompas M. Is a ventilator-associated pneumonia rate of zero really possible? Curr Opin Infect Dis 2012; 25(2): 176-82.

15. Bouadma L, Mourvillier B, Deiler V, Le Corre B, Lolom I, Régnier B, et al. A multifaceted program to prevent ventilator-associated pneumonia: impact on compliance with preventive measures. Crit Care Med 2010; 38(3): 789-96.

16. Leasure a R, Stirlen J, Lu SH. Prevention of Ventilator-Associated Pneumonia Through Aspiration of Subglottic Secretions: A Systematic Review and MetaAnalysis. Dimens Crit Care Nurs 2012; 31(2): 102-17.

17. Frost S a, Azeem A, Alexandrou E, Tam V, Murphy JK, Hunt L, et al. Subglottic secretion drainage for preventing ventilator associated pneumonia: A meta-analysis. Aust Crit Care 2013; 26(4): 180-188;

18. Kelley SD. Number needed to treat for subglottic secretion drainage technology as a ventilator-associated pneumonia prevention strategy. Crit Care 2012; 16(5): 446

19. Souza CR De, Santana VTS. Impact of supra-cuff suction on ventilator-associated pneumonia prevention. Rev Bras Ter intensiva 2012; 24(4): 401-6. 